\title{
Radar and optical leonids
}

\author{
N. Brosch ${ }^{1}$, D. Polishook ${ }^{1}$, R. Helled ${ }^{1}$, S. Schijvarg ${ }^{1}$, and M. Rosenkrantz ${ }^{2}$ \\ ${ }^{1}$ Beverly and Raymond Sackler Faculty of Exact Sciences, Tel Aviv University, Tel Aviv 69978, Israel \\ ${ }^{2}$ Radar Division, ELTA Ltd., POB 330, Ashdod 77102, Israel
}

Received: 25 September 2003 - Published in Atmos. Chem. Phys. Discuss.: 9 March 2004

Revised: 16 June 2004 - Accepted: 20 June 2004 - Published: 6 July 2004

\begin{abstract}
We present joint optical-radar observations of meteors collected near the peak of the Leonid activity in 2002. We show four examples of joint detections with a large, phased array L-band radar and with intensified video cameras. The general characteristic of the radar-detected optical meteors is that they show the radar detection below the termination of the optical meteor. Therefore, at least some radar events associated with meteor activity are neither head echoes nor trail echoes, but probably indicate the formation of "charged clouds" after the visual meteor is extinguished.
\end{abstract}

\section{Introduction}

Since the early days of radar operations, echoes at high altitude were identified with meteor activity. While most of the observations reported in the literature relate to observations at frequencies of tens of $\mathrm{MHz}$, a few reports deal with higher frequencies, at $400 \mathrm{MHz}$ or higher. In particular, the Arecibo and EISCAT radars were used successfully for such purposes (e.g. Janches et al., 2000; Pellinen-Wannberg, 2001). These are large parabolic reflectors, directing a high-power, narrowangle beam towards the sky. Meteoroids entering the beam produce radar echoes; these can, in principle, be of two kinds. The more obvious, but hard to detect, is the 'head echo', produced by the immediate shock front and/or by the plasma created near the meteoroid itself. The more common echo is from the plasma cloud that forms near and behind the meteoroid; this 'trail echo' is detected also in HF and VHF bands. The meteor itself is, in most cases, not detected at all.

The optical signature of a meteoroid, the luminous headand-tail structure, is classically thought to be produced by the ablation of the meteoroid followed by the ionization of the nearby atmosphere. This is mainly a secondary effect; the meteoroid material melts and its droplets are ejected into the

Correspondence to: N. Brosch

(nbrosch@yahoo.com) atmosphere producing the observed ionization pattern (Baggaley, 2002). The ionization is produced in two regions: an approximately spherical volume with a radius of a few meters enclosing the meteoroid where the head echo is produced, and a column of ionization, formed by $\sim 10$ collisions of each atom ablated from the meteoroid with atmospheric atoms, that can be distributed along $\sim 10 \mathrm{~km}$ and which produces the trail echo. The value of $\sim 10$ collisions results from an estimate of the velocity of an ablated atom off a Leonid meteor, and follows Bronshten (1983).

Grygar et al. (1968) and Kohoutek et al. (1970) studied the association of optical meteors with radar echoes. They used in 1962 the Ondrejov radar at $37.5 \mathrm{MHz}$ in conjunction with visual observations and searched for radar returns within $\Delta t= \pm 20 \mathrm{~s}$ of the optical meteor. Grygar et al. found that all bright meteors, with absolute magnitude brighter than $0 \mathrm{mag}$, had radar counterparts, but that only $12 \%$ of the fainter meteors, between +1 and +3 mag, were recorded by the radar. None of the telescopic meteors they observed had radar counterparts.

Znojil et al. (1985) analyzed Ondrejov radar observations of meteors performed in conjunction with visual telescopic observations in 1972-1973. The identification of correlated events was based on agreement of time and range. Out of 283 meteors, they identified 65 as joint events. Znojil et al. found that meteors observed at low elongation angles from the shower radiant had the radar echo following by a fraction of a second the optical detection.

Jones and Webster (1991) analyzed visual observations together with radar returns from the Springfield installation operating at $33 \mathrm{MHz}$ and accepted optical-radar associations if $\Delta \mathrm{t}= \pm 1 \mathrm{~s}$ and if the approximate positions of the radar echo and of the visual sighting of the optical meteor coincided. They found that more than half of the bright meteors $(\mathrm{M}<0 \mathrm{mag})$ produced head echoes and that this fraction decreased with the meteor brightness. 
An attempt to correlate optical meteor detections obtained with two-station ISIT cameras and echoes from the EISCAT meteor radar during the 1990 Geminids was reported by Pellinen-Wannberg et al. (1998). Despite using a large UHF $(930 \mathrm{MHz})$ radar with a detection rate of $\sim 65$ echoes per hour, and detecting 59 optical meteors with one station and 35 with the other during $\sim 35 \mathrm{~h}$, no coincident opticalradar meteor was identified. Pellinen-Wannberg et al. explain the lack of correlated events by the different sensitivities of the systems they used: while the optical cameras had very large fields of view but were sensitive only to meteors brighter than +4 mag, the EISCAT radar detected meteors brighter than $+10 \mathrm{mag}$, but only those that passed through a $\sim 1 \mathrm{~km}$-wide column along the radar beam. The lack of correlations would therefore be caused by low statistics of bright meteors.

Pecina et al. (2001) reported simultaneous double-station TV and radar observations of Perseid meteors performed at the Ondrejov observatory. Out of 199 meteors they selected 19 that were observed by both cameras and the radar and had a similar range. They attempted to redefine the relation between the absolute magnitude of a meteor and the electron line density it produced, but could not fit the observations with a single relation.

Dyrud et al. (2002) described multi-frequency meteor observations performed with the ALTAIR tracking radar at the Reagan test site in the Kwajalein atoll. They proposed that one could discern three regimes when explaining radar echoes from meteors. One is the head echo formed by the plasma immediately adjacent to the ablating meteor. The second is a region adjacent to the head, from which no echoes are received and is explained by "laminar" plasma flow from the head. The smooth plasma flow directs the electromagnetic energy away from the radar beam and prevents echoes from being returned to the radar antenna, creating essentially "stealthy" conditions. The third regime is encountered in the trail, where echoes form off the turbulence in the plasma. Close et al. (2004) and Hunt et al. (2004) published some aspects of this work.

Recently, Sato (2003) reported successful joint opticalradar observations with the MU radar at $46.5 \mathrm{MHz}$ and with intensified video cameras. Out of almost 700 echoes collected during $27.7 \mathrm{~h}$ of observation, spread over 14 nights in different months of 2002, they identified 44 optical events synchronized with the radar where the optical meteor track matched almost perfectly that of the radar.

During the recent Leonid storms we observed from Israel optical and radar signatures of meteoric activity. The radar system we used is an operational L-band phased array installation, characterized by (a) large output power, (b) wide sky coverage by fast electronic scanning with a synthesized beam, and (c) unambiguous detections, free of range doubling and of other instrumental artifacts. These indicate that the expected results from this installation could be superior to those from EISCAT and, at least regarding the issue of sky area coverage, would surpass those from the Arecibo radar, despite the reduction in detection efficiency due to the frequency dependence of the plasma reflection coefficient.

Our previous result from radar meteor campaigns relates to the altitude distribution of the radar echoes in the L-band at $\sim 1 \mathrm{GHz}$ (Brosch et al., 2001; Schijvarg et al., 2003). This is the discovery of two populations of echoes, one corresponding to the classical region of meteoroid ablation at 80$130 \mathrm{~km}$ (class I) and the other at much higher altitudes, peaking at $\sim 250 \mathrm{~km}$ (class II). In both cases it is clear that the radar echo is from plasma (mostly electrons) with significant radar cross section.

The reality of the high altitude radar echoes peak was questioned and one of the objections to our first reports was that it is not clear whether it is the optical meteors that are producing the L-band radar echoes or perhaps some other phenomenon. In this paper we present preliminary results from a joint radar-optical campaign to observe meteors conducted in Israel during the 2002 Leonid storm. We first describe the observations, and then show a few examples of optical meteors that correspond to radar echoes' detections. Further examples will be shown in subsequent papers. The results show unequivocally the production of L-band radar returns from optical meteors.

\section{Observations}

We report on joint radar and optical observations during part of the night of 18-19 November 2002, prior to the predicted first 'storm' peak of the Leonid shower at 04:03 UT. The radar observations were performed with a large phased array system operating in the L-band. As this is an operational system, we are not at liberty to discuss many details about this classified system, its operation, and its detection of meteors. The advantage of this system, as already mentioned above and in comparison with other radars used for meteor observations, is the ability to scan a large sky volume quasi-simultaneously by electronically steering the synthesized beam. For the observations reported here the bore axis of the radar was oriented almost due east at an altitude of $\sim 30^{\circ}$, and the projected sky area surveyed with the scanning beam covered approximately one steradian. The borders of the scanned atmospheric area, in the topocentric frame of reference of the Wise Observatory in Mizpe Ramon where the ICCDs were operated, were $\sim 16^{\circ}$ to $\sim 47^{\circ}$ in elevation, $\sim 23^{\circ}$ to $\sim 137^{\circ}$ in azimuth, and the search was limited to a height range of $\sim 52$ to $\sim 214 \mathrm{~km}$. The total sky area searched for radar echo events was thus a bit more than one steradian.

The radar detections consist of indications of elevation, azimuth to within a fraction of a degree, and range to within a fraction of a $\mathrm{km}$, for directions from which the echoes were received. A GPS-derived UT time stamp identifies these detections, which are good to about one second or better. We stress that "detection" here means a combination of a number 
of transmitted pulses, with appropriate coding, and the reception of their echoes.

The optical observations were collected with intensified CCD (ICCD) video systems described previously (Brosch et al., 2002). The only difference with the previously reported campaigns was the use of 50-mm f/0.95 lenses. These yielded fields of view of $6^{\circ} \times 8^{\circ}$ with a scale of $\sim 1$ arcmin per pixel that show stars as faint as $\sim 8 \mathrm{mag}$. The cameras were oriented with their axes toward azimuth 050 and were pointed to elevations of $\sim 27^{\circ}$ and $\sim 24^{\circ}$ above the local horizon. In this direction, the cameras viewed the atmosphere above the town of Mizpe Ramon, some five km away from the Wise Observatory. To allow improved observing conditions, the city lights of Mizpe Ramon were turned off for this observation.

The video rates used by the ICCDs were PAL-standard (50 odd-even interlaced frames per second), the video capture was done in real time using Meteor II cards with the MetRec software (Molau, 2001), and the images were recorded in parallel on $\mathrm{Hi} 8$ digital tapes. The data collection used a GPS-derived UT time stamp, similar to the radar observations, thus the two systems were on the same time base. The only difference was that the ICCD systems were manually synchronized to the GPS time, yielding a timing jitter of $\sim 1 \mathrm{~s}$.

Due to the imperfect alignment of the cameras, the pointing of the lower one (W2), was rotated by $\sim 6^{\circ}$ relative to the camera aimed at the higher altitude (W1). The sky areas imaged by the two systems overlapped by $\sim 2^{\circ}$, allowing a total sky region of $\sim 70$ square degrees to be imaged. Although the atmospheric volume common to the cameras and the radar system was relatively small, as only a small fraction of the radar-surveyed area was imaged by the cameras, we managed to collect a number of events that were common to both systems.

The search for radar-optical common events was conducted primarily using temporal coincidence of the optical and the radar detections, as both systems used GPS timing, followed by a check that the radar detection was located in the common atmospheric volume, or close to the extrapolated trajectory of the optical meteor, or in its vicinity. We are aware that this procedure can miss some common events. For instance, if the radar-reflecting plasma is formed at some stage of the meteor process but is fast drifting off the optical track, it is possible that the radar detection would be found quite some way off the optical detection. On the other hand, during the counterpart search we allow for the possibility that the radar-reflecting plasma would be decoupled from the optical meteor by looking for time-correlated radar events localized in a region smaller than the entire area covered by the radar scans, but larger than the area covered by the imagers.

\section{Results}

The sample of optical meteors considered here was restricted to the one-hour time period from 02:00 to 03:00 UT on 19 November. During this period the W1 camera recorded 22 meteors and W2 recorded 17 meteors. Of these 39 meteors, eight were common to both cameras and are further selected here as a two-camera sub-sample. The radar system yielded 544 detections during the period from 02:07:10 UT to $03: 11: 03 \mathrm{UT}$, or a rate of 510.9 detections per hour.

The search for common radar-optical events included 33 radar detections that had $\sim$ simultaneous optical meteor detection (within $10 \mathrm{~s}$ ) and were located approximately in the vicinity of the optical meteor, as described below. From these 33 radar events we selected for further analysis a smaller number that were detected in both cameras. Four such events are described below and the combined images recorded by the video cameras are shown in Figs. 1a to 4a. We stress here that our original data set includes more events, but these were recorded by a single ICCD system only, or during other time intervals when both systems operated. The full data set will be discussed in a subsequent paper.

We calculated the topocentric track of each selected meteor for each beginning and end of track on the individual video frames of its appearance. The astrometry was performed via five to seven neighborhood stars that were identified and measured on the combined frames (shown below). The plot of the track, in topocentric coordinates, was compared with the location of the radar echo, as shown in the four figures below.

\section{Interpretation}

We presented here four examples of Leonid meteors collected during the 2002 campaign at the Wise Observatory to which we attempted to associate radar detections from a high-power L-band phased array system. Three of the four examples are of bright meteors that showed flaring near their terminations, but one does not show such flaring. That meteor, observed at 02:42:31 UT, did show a peculiar intermediate dimming instead of flaring.

We first estimate the chance of mistakenly associating optical with radar events. Following Kohoutek et al. (1970), we define $f_{\text {opt }}$ and $f_{\text {rad }}$ to be the apparent rate of detections by the optical and radar systems, respectively. We also define $F$ to be the ratio of sky areas covered by the search for radar counterparts connected with optical detections (some $20 \times 20^{\circ}$ ), and the full area covered by the radar systems. For the observation described here $F \approx 400$ square degrees (optical)/3283 square degrees $($ radar $)=0.12$. As we selected a time interval of $\pm 10 \mathrm{~s}$ around each optical meteor (180 such intervals in one hour) to search for radar counterparts, the expected number of random coincidences in the period from 02:00 UT to 03:00 UT is $n_{\text {rand }}=f_{\text {opt }} \times f_{\text {rad }} \times F / 180$. 


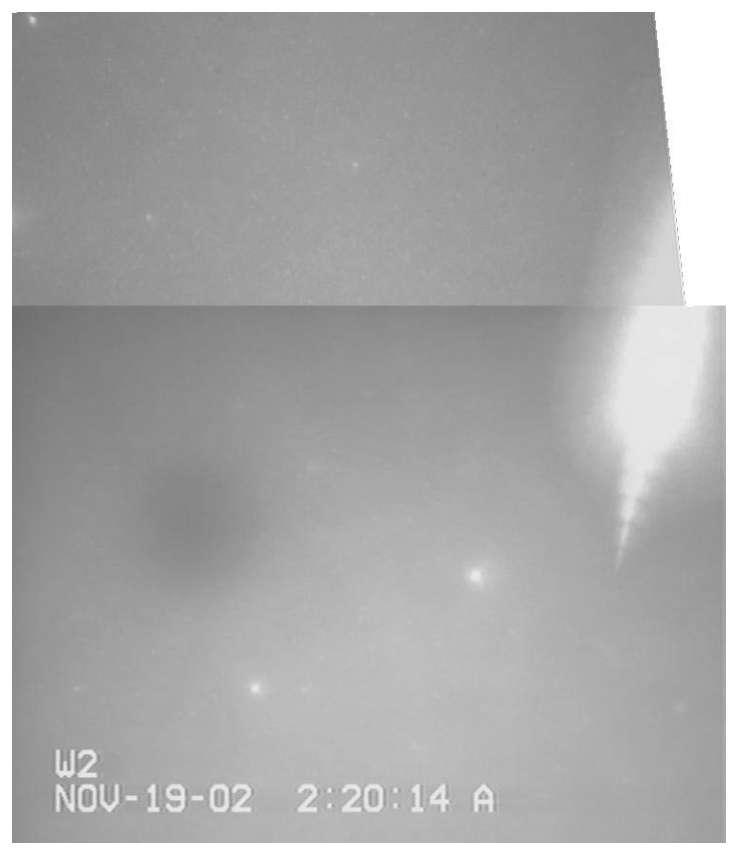

(a)

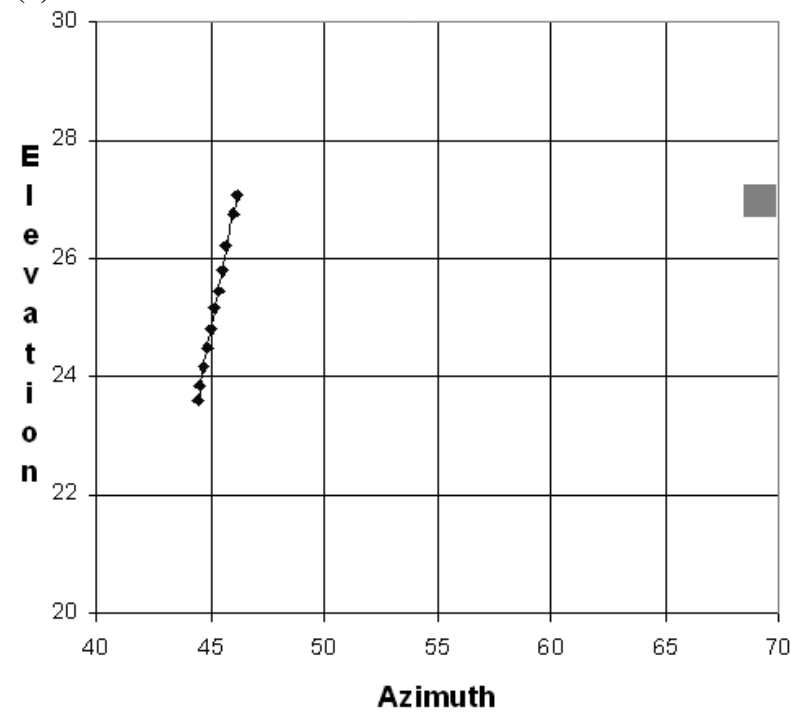

Fig. 1. (a) Combined image of meteor at 02:20:10 UT. The image was produced by combining the meteor records from each of the ICCDs (W1 and W2), after compensation for the slight rotation of one FOV relative to the other. This meteor produced a significant flare but it did not terminate then, rather continuing for $\sim 200 \mathrm{~ms}$ after the end of the flare. (b) Plot of the meteor track from (a) with the indication of the location of the radar echo associated with this meteor and detected at Azimuth=69, Elevation=27, Height $=113 \mathrm{~km}$, Time $=02: 19: 59$ UT, plotted here as the gray box.

As explained above, $f_{\text {opt }}=8$ and $f_{\text {rad }}=510.9$, yielding $\sim 3$ chance associations expected during one hour of joint optical and radar observations. As we detected eight common events, we can accept with a reasonably high level of confidence the association of the four optical-radar events ana-

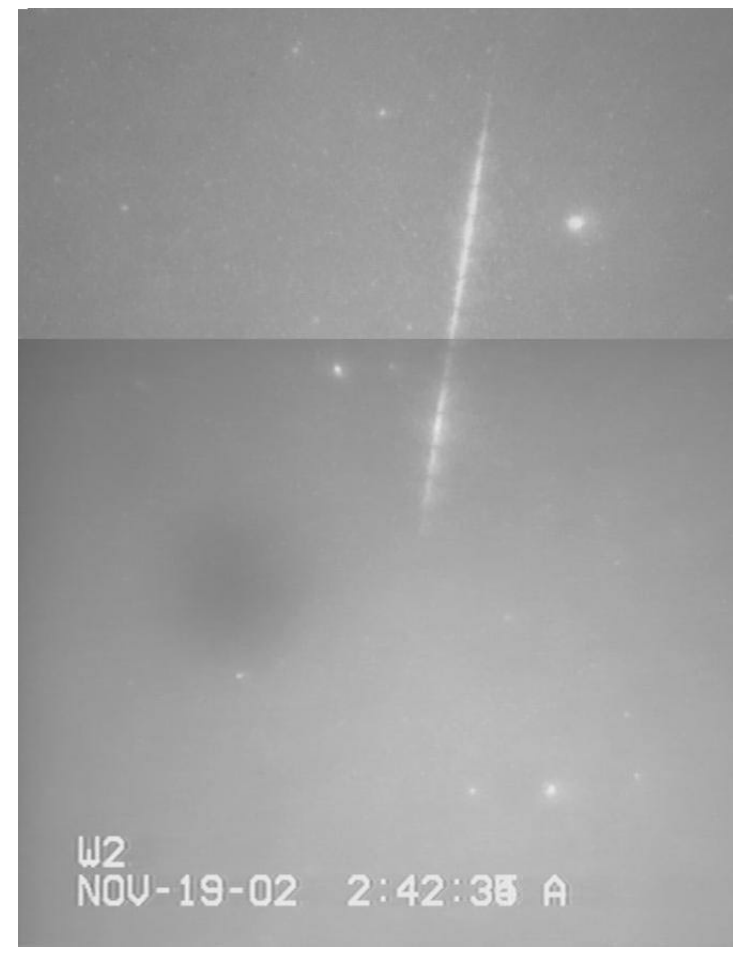

(a) (b)

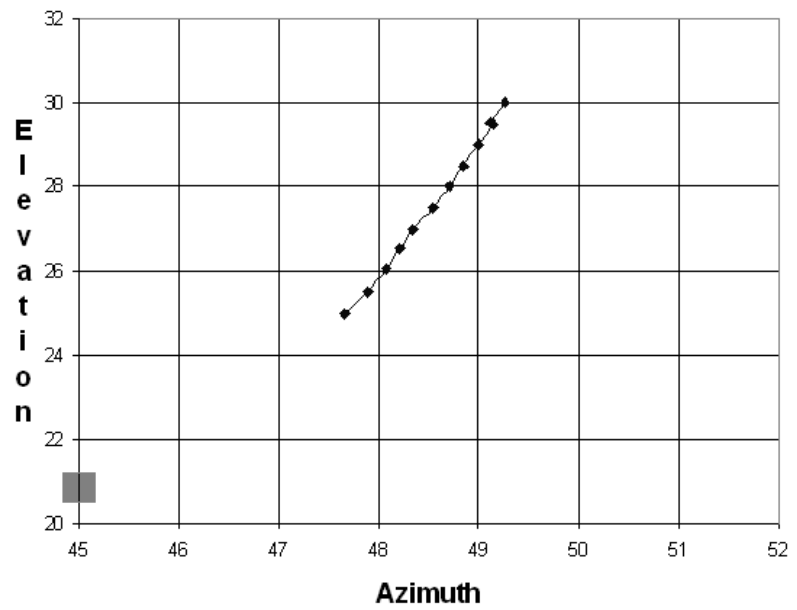

Fig. 2. (a) Combined image of meteor at 02:42:31 UT. This meteor did not flare strongly, but showed a distinct dimming near the middle of its track. Although shown here at the interface between the two FOVs, the dimming is clearly visible in the record from the W1 camera, which contains the entire track. (b) Optical track of the meteor shown in (a) and of the radar detection at Azimuth $=45$, Elevation $=21$, Height $=111 \mathrm{~km}$, Time $=02: 42: 30$ UT, shown here as the gray square. A second radar detection at Azimuth=59, Elevation=21 and detected seven seconds after the optical meteor, is not shown here.

lyzed here. We note that it is likely some of the associations are false, but emphasize that it is unlikely to have all of them attributable to chance. 


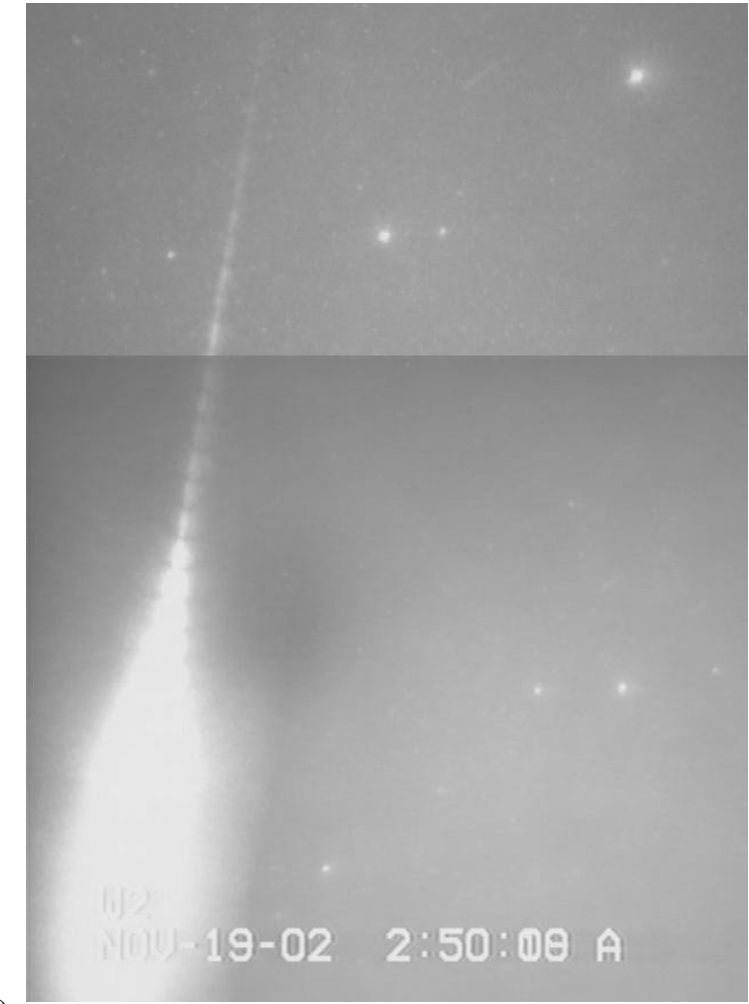

(a)

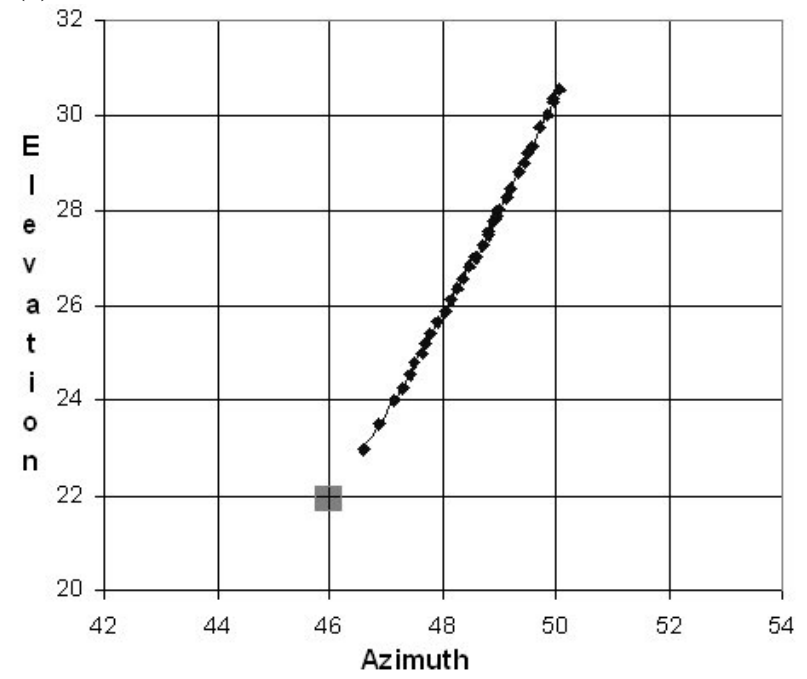

Fig. 3. (a) Combined image of meteor at 02:50:06 UT. This meteor produced a strong flare, although from the ICCD images it is not clear whether this was a terminal flare or the meteor continued after the flare, as did the one shown in Fig. 1. (b) Optical track detection associated with the meteor shown in (a). The radar detection was at Azimuth=46, Elevation=22, Height $=72 \mathrm{~km}$, Time=02:50:09 UT and is marked here by the gray square.

The assumption here is that both systems, radar and optical, viewed continuously the sky region they patrolled. However, as explained above, the radar beam was scanning the area, not staring at one location. The requirement, therefore, translates into the assumption that the lifetime of a radar echo

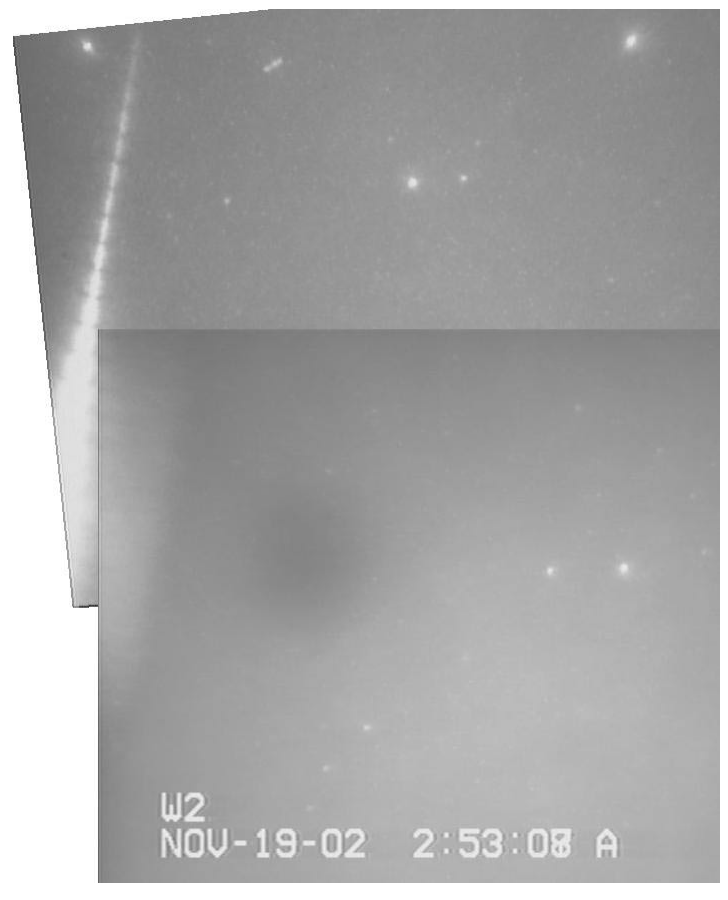

(a)

(b)

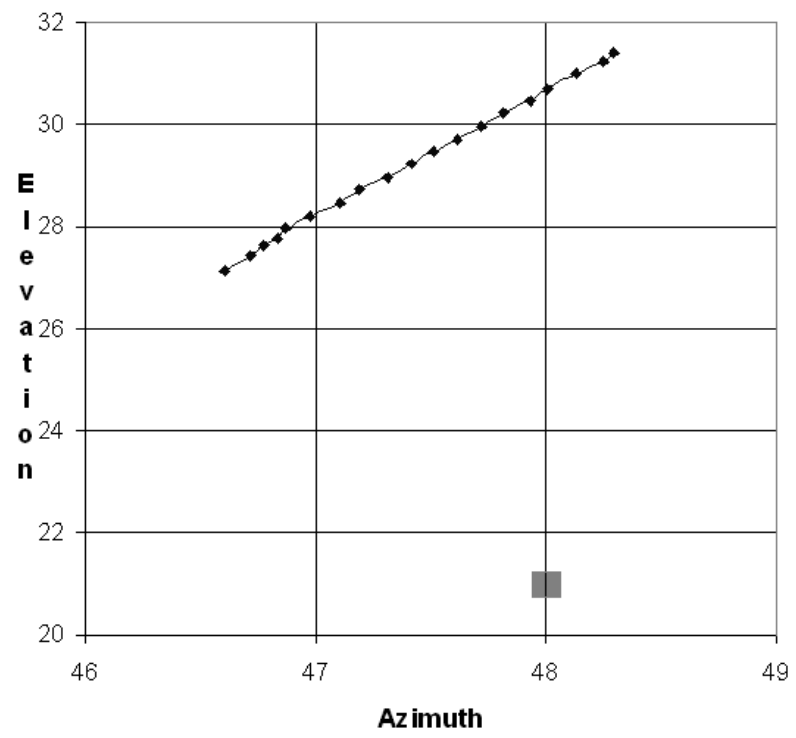

Fig. 4. (a) Combined image of meteor at 02:53:03 UT. This meteor terminated out of the FOV of W1 and W2, but from the partial record of $\mathrm{W} 2$ it seems that the flare continued below the recorded altitude. (b) Optical track and associated radar detection for meteor shown in (a). The distant radar detection, shown here as the dark square, was at Azimuth $=48$, Elevation=21, Height $=67 \mathrm{~km}$, Time $=02: 52: 53$ UT and we estimate this association to be less probable than in the three other cases, because the time difference puts the radar detection ten seconds earlier than the optical.

be longer than the time between successive sweeps of the beam. As far as we are aware, this is indeed the case but for reasons discussed above we cannot quote the duration of the sweep. In other instances, we may lose some radar echoes that would dissipate before being illuminated by the beam. 


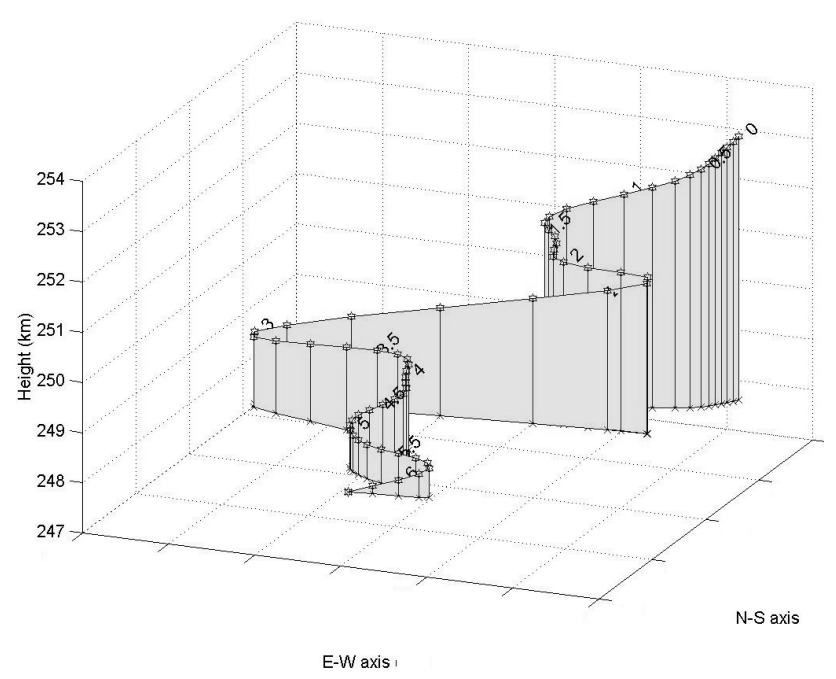

Fig. 5. Radar event detected in Israel on 30 June 1999 during the $\beta$ Taurid meteor shower.

The common characteristic noted here is that in all cases the radar counterpart was detected below the optical meteor, at least below its track beginning, and significantly distant from it. While in few cases this may be due to a chance coincidence, with the radar echo unrelated to the optical meteor, the 'best case' example, of the meteor at 02:42:31 UT (Figs. 3a and b), has the radar echo received simultaneously with the optical detection (within the timing accuracy) but appearing below and on the downward extension of the optical track.

This finding does not correspond to the accepted mechanism of either head or trail radar echoes because in both cases the radar return is obtained from the visual meteor itself. There is no possibility to receive a radar return if no plasma is generated and classically, this process accompanies that of the light production. In other words, "there is no smoke without a fire".

Jones et al. (1999) modeled the plasma in the head echo as a quasi-spherical region some $1-2 \mathrm{~m}$ in radius. Boyd (2000) presented similar results from numerical simulations of Leonid meteors. Observations reported during the 2002 Leonid shower, and collected with the high frame-rate camera of the University of Alaska-Fairbanks seem to indicate that the region may extend as far back as tens of meteors from the head. These models imply that the head echo must be associated with the dense plasma formed immediately next to the meteoroid and does probably follow the development of the light emission. By inference, when the light production from the meteor is extinguished the plasma must disappear and the radar return must vanish.

How can we therefore explain our observation that the meteor radar echo is consistently received a significant distance from the optical meteor? There are at least two possibilities, each invoked in the past to explain other properties of radar meteors. One is the production of ionization through chemi- cal reactions in the meteor trail that do not produce copious amounts of light and the other is the production of 'meteor smoke', some kind of electrically charged aerosol. In both cases one could achieve consistent radar returns without associated light production and both mechanisms may be relevant.

The example of ionic reactions is presented, e.g. by Baggaley (2002), who invokes ion oxide formation from meteoric atomic ions and ozone and, at altitudes below $90 \mathrm{~km}$ the formation of ion oxides through a three-body charge exchange process. The lifetimes of electrons released by chemical loss ranges from $3 \mathrm{~s}$ at $80 \mathrm{~km}$ to $200 \mathrm{~s}$ at $100 \mathrm{~km}$. The release of ions at high altitudes, followed by chemical reactions when the ions descend below $100 \mathrm{~km}$, has been proposed previously by Murad (2001). The question is whether it is reasonable to assume that the meteoric atomic ions might travel a considerable way from their release until they produce the plasma that is detected by radar.

The other possibility, of charged meteoric dust, was put forward by Kelley et al. (1998) to explain a unique observation of a radar meteor detection, coincident with an insitu measurement of electrons in a meteor trail by a Super Arcas sounding rocket launched from Alaska in 1983. The data indicate an increase of the electron density at $92.3 \mathrm{~km}$, from an ambient value of $\sim 2.3 \times 10^{4}$ electrons $\mathrm{cm}^{-3}$ to $\sim 3.9 \times 10^{4}$ electrons $\mathrm{cm}^{-3}$ for a distance of $42 \mathrm{~m}$ and with extremely sharp edges, where the electron density gradient was $10^{3}$ electrons per meter. The $50 \mathrm{MHz}$ Poker Flats radar showed a reflection at the altitude of the electron enhancement that lasted for some six minutes and drifted within the radar beam with a velocity of a few meters per second.

Kelley et al. (1998) interpreted this unique observation as indicating the formation of a dusty trail of $\sim 50 \mathrm{~nm}$ particles, carrying on average a charge of one electron per particle. These particles would fall freely and would be carried by the high altitude winds, but their diffusion coefficient would be much lower than that of atmospheric ions because of their considerable mass.

If the hypothesis of charged meteoric 'smoke' particles can be sustained, this would explain why "plasma" could produce radar echoes even though the meteor itself is no longer visible. Such a hypothesis would also explain some puzzling observations obtained by us with the same radar during other meteor showers, as well as possibly some of the phenomena associated with persistent trains. These observations show some cases where the radar echo proceeded with a space velocity of a few $\mathrm{km} / \mathrm{sec}$, and where the tracked radar echo changed direction, sometimes even ascending to higher altitudes.

Figure 5 presents an example of a radar detection obtained in 1999 during the daytime $\beta$ Taurid meteor shower with the same radar equipment used here and displayed as a three-dimensional plot. The plot shows the progression of high altitude radar echoes during a period of six seconds. Individual detections are marked on the plot and a time tag is 
added every $0.5 \mathrm{~s}$. The ticks on the E-W axis are spaced by one $\mathrm{km}$; those on the $\mathrm{N}-\mathrm{S}$ axis are spaced by five $\mathrm{km}$. This choice exaggerates the sinuous motion of the echoes.

The radar echoes indicate a slow-moving radar reflector that traces a sinuous path (accentuated here by the different $\mathrm{N}-\mathrm{S}$ and E-W axes) at very high altitude. A charged mass of dust grains, with a low diffusion coefficient and carried by high-altitude winds, would probably show such a behavior. We note that more than one such long-duration echo was recorded by our systems, implying that this is not an extremely rare phenomenon.

The fragmentation of meteors at very high altitudes (220$260 \mathrm{~km}$ ) was one of the interesting results of CampbellBrown and Jones (2003) when modeling Geminid dustballs in the context of the "initial radius" problem. As the general consensus is that Leonids are among the more fragile of meteors, it seems likely that a similar fragmentation process could take place for them. This would produce, in some cases, clouds of dust that may be electrically charged and could reflect radar emission.

\section{Conclusions}

We presented four examples of optical meteors that were associated with radar echoes. The optical information was obtained with visible-light intensified video systems operating at the Wise Observatory, Israel, and the radar data were collected by a large phased-array radar system operating in the L-band. The radar allowed the monitoring of a large volume of space and part of it overlapped with the sky region observed by the video systems. The main characteristic of the observations is that the associated radar echo appears consistently below the optical meteor. We pose the hypothesis that, at least in these cases, a cloud of charged particles (meteoric ions or dust) proceeds to lower altitudes after the optical meteor is extinguished.

Acknowledgements. Observations of meteors in Israel are supported by a grant from the Israel Science Foundation. We are grateful to ELTA, Ltd., for allowing the use of its facilities to reduce the radar observations. N. Brosch acknowledges support from the Austrian Friends of Tel Aviv University. We are grateful to the former Mayor of the town of Mizpe Ramon, D. Dvash, and to the city council, for turning off the city lights during the 2002 Leonid shower.

Edited by: D. Janches

\section{References}

Baggaley, J.: Radar Observations, in "Meteors in the Earth's Atmosphere", edited by Murad, E. and Williams, I. P., Cambridge (UK), Cambridge University Press, 123, 2002.

Boyd, I. D.: Computation of Atmospheric Entry Flow about a Leonid Meteoroid, in "Leonid Storm Research", edited by Jenniskens, P., Rietmeijer, F., Brosch, N., and Fonda, M., Dordrecht, Kluwer Academic Publishers, 93, 2000.
Bronshten, V. A.: Physics of meteoric phenomena, Dordrecht, Kluwer Academic Publishers, 215, 1983.

Brosch, N., Schijvarg, L. S., Podolak, M., and Rosenkrantz, M. R.: Meteor Observations from Israel, in "Meteoroids 2001", ESA SP-495, 165, 2001.

Campbell-Brown, M. and Jones, J.: Determining the initial radius of meteoric trains: fragmentation, Monthly Notices of the Royal Astronomical Society, 343, 775, 2003.

Close, S., Oppenheim, M., Hunt, S., and Coster, A.: A technique for calculating plasma density and meteoroid mass from radar head echo scattering, Icarus, 168, 43, 2004.

Dyrud, L. P., Oppenheim, M. M., Close, S., and Hunt, S.: Interpretation of non-specular radar meteor trails, Geophys. Res. Lett., 29, (21), 8-1, 2002.

Grygar, J., Kohoutek, L., and Plavcova, Z.: Simultaneous Radar and Optical Observations of Meteors at Ondrejov, in "Physics and Dynamics of Meteors", IAU Symposium no. 33, edited by Kresak, L. and Millman, P. M., 63, 1968.

Hunt, S. M., Oppenheim, M., Close, S., Brown, P. G., McKeen, F., and Minardi, M.: Determination of the meteoroid velocity distribution at the Earth using high-gain radar, Icarus, 168, 34, 2004.

Janches, D., Mathews, J. D., Meisel, D. D., and Zhou, Q.-H.: Micrometeor Observations Using the Arecibo $430 \mathrm{MHz}$ Radar, I. Determination of the Ballistic Parameter from Measured Doppler Velocity and Deceleration Results, Icarus, 145, 53, 2000.

Jones, J., Jones, W., and Halliday, I.: The Head Echo Problem a Solution at Last?, in "Meteoroids 1998", edited by Baggaley, W. J. and Porubcan, V., Bratislava, Astronomical Institute of the Slovak Academy of Sciences, 29, 1999.

Jones, J. and Webster, A. R.: Visual and Radar Studies of Meteor Head Echoes, Planet. Space Sci., 39, 873, 1991.

Kelley, M. C., Alcala, C., and Cho, J. Y. N.: Detection of a Meteor Contrail and Meteoric Dust in the Earth's Upper Mesosphere, J. Atmos. S-P, 60, (3), 359, 1998.

Kohoutek, L., Grygar, J., Plavcova, Z., and Kvizova, J.: Comparison of Radar and Optical Meteor Observations, Bull. Astron. Inst. Czechosl. 21, 18, 1970.

Molau, S.: The AKM video meteor network, in "Meteoroids 2001", ESA SP-495, 315, 2001.

Murad, E.: Physics and Chemistry of Meteoroids in the Upper Atmosphere, in "Meteoroids 2001", ESA SP-495, 229, 2001.

Pecina, P., Koten, P., Stork, R., Pridal, P., and Novakova, D.: Relation between the Optical and Radar Characteristics of Meteors: Perseids 1998 and 1999, in "Meteoroids 2001", ESA SP-495, 399, 2001

Pellinen-Wannberg, A.: The High Power Large Aperture Radar Method for Meteor Observations, in "Meteoroids 2001", ESA SP-495, 443, 2001.

Pellinen-Wannberg, A., Westman, A., Wannberg, G., and Kaila, K.: Meteor Fluxes and Visual Magnitudes from EISCAT Radar Event Rates: a Comparison with Cross-section Based Magnitude Estimates and Optical Data, Ann. Geophys., 116, 1475, 1998.

Sato, T.: Parameter estimation of meteors from radar head echoes combined with optical observations, Radar Meteor Workshop, Arecibo, 2003.

Znojil, V., Hollan, J., and Simek, M.: The Relation Between Meteor Optical Brightness and Properties of the Ionized Trail, Bull. Astron. Inst. Czechosl., 36, 44, 1985. 